\title{
Magnetic ground state and spin fluctuations in MnGe chiral magnet as studied by muon spin rotation
}

\author{
N. Martin, ${ }^{1}$ M. Deutsch, ${ }^{2}$ F. Bert, ${ }^{3}$ D. Andreica, ${ }^{4}$ A. Amato, ${ }^{5}$ P. Bonfà, ${ }^{6}$ R. De Renzi, ${ }^{6}$ U. K. Rößler, ${ }^{7}$ P. Bonville, ${ }^{8}$ \\ L. N. Fomicheva, ${ }^{9}$ A. V. Tsvyashchenko, ${ }^{9,10}$ and I. Mirebeau ${ }^{1, *}$ \\ ${ }^{1}$ Laboratoire Léon Brillouin, CEA, CNRS, Université Paris-Saclay, CEA Saclay 91191 Gif-sur-Yvette, France \\ ${ }^{2}$ Université de Lorraine, Laboratoire CRM2,UMR UL-CNRS 7036, 54506 Vandoeuvre-les-Nancy, France \\ ${ }^{3}$ Laboratoire de Physique du Solide, UMR CNRS 8502, Université Paris Sud, FR-91140 Orsay, France \\ ${ }^{4}$ Faculty of Physics, Babes-Bolyai University, 400084 Cluj-Napoca, Romania \\ ${ }^{5}$ Laboratory for Muon Spin Spectroscopy, PSI, CH-5232 Villigen PSI, Switzerland \\ ${ }^{6}$ Dipartimento di Fisica e Scienze della Terra and Unità CNISM di Parma, Università di Parma, 43124 Parma, Italy \\ ${ }^{7}$ Leibnitz Institute for Solid State and Material Research, IFW Dresden D-01069, Germany \\ ${ }^{8}$ SPEC, CEA, CNRS, Université Paris-Saclay, CEA-Saclay, 91191 Gif-sur-Yvette, France \\ ${ }^{9}$ Vereshchagin Institute for High Pressure Physics, Russian Academy of Sciences, 142190 Troitsk, Moscow, Russia \\ ${ }^{10}$ Skobeltsyn Institute of Nuclear Physics, MSU, Vorob'evy Gory 1/2, 119991 Moscow, Russia \\ (Received 2 February 2016; revised manuscript received 19 April 2016; published 5 May 2016)
}

\begin{abstract}
We have studied by muon spin resonance ( $\mu \mathrm{SR}$ ) the helical ground state and fluctuating chiral phase recently observed in the MnGe chiral magnet. At low temperature, the muon polarization shows double-period oscillations at short-time scales. Their analysis, akin to that recently developed for MnSi [A. Amato et al., Phys. Rev. B 89, 184425 (2014)], provides an estimation of the field distribution induced by the Mn helical order at the muon site. The refined muon position agrees nicely with ab initio calculations. With increasing temperature, an inhomogeneous fluctuating chiral phase sets in, characterized by two well-separated frequency ranges which coexist in the sample. Rapid and slow fluctuations, respectively, associated with short-range and long-range ordered helices, coexist in a large temperature range below $T_{\mathrm{N}}=170 \mathrm{~K}$. We discuss the results with respect to $\mathrm{MnSi}$, taking the short helical period, metastable quenched state, and peculiar band structure of MnGe into account.
\end{abstract}

DOI: 10.1103/PhysRevB.93.174405

\section{INTRODUCTION}

Spin fluctuations in itinerant systems have attracted strong attention since the pioneering work of Moriya [1], providing a unified theory for the Curie-Weiss dependence of the spin susceptibility. Such fluctuations, either thermal or quantum, are usually a precursor of a transition towards a magnetically ordered ground state. In critical phenomena, low-energy fluctuations of the order parameter, extending over increasing length scales, yield a second-order transition obeying Ginzburg-Landau universality laws. Such a secondorder phase transition could be avoided if the fluctuations are strong enough, with the system evading the associated entropy by undergoing a first-order transition without any divergence of the correlation length.

In B20 itinerant chiral magnets such as $\mathrm{MnSi}$, FeGe, or $\mathrm{MnGe}$, the crucial role of the spin fluctuations appears already in the ground state, when applied pressure induces a first-order quantum transition from the ordered helical state to a non-Fermi-liquid state with partial magnetic order, involving chiral fluctuations of local magnetic moments [2-5]. At finite temperature and under magnetic field, an intermediate chiral phase (also called fluctuation disordered regime) is stabilized between the ordered and paramagnetic phases. The nature of this phase has been discussed in the literature. One explanation invokes chiral mesophases in analogy with

\footnotetext{
*isabelle.mirebeau@cea.fr
}

chiral nematics, including disordered liquid phases composed of skyrmionic double-twisted or multiply twisted spin textures [4-7]. Another scenario assumes fluctuating helices [8,9] with finite length scale, isotropically distributed in space, and a fluctuation-induced first-order transition [10] as explained by Brazovskii universality [11]. The chiral fluctuations could be the source of soft modes, stabilizing a skyrmion lattice phase in $\mathrm{MnSi}$ and $\mathrm{FeGe}$ just below the ordering transition $[3,8,12]$.

In this series, MnGe stands out as a highly topical magnet, still poorly understood. Synthesized under high pressure and temperature [13], MnGe exists in a metastable and powdered state only. The strong exchange interaction yields a high transition temperature $\left(T_{\mathrm{N}}=170 \mathrm{~K}\right)$ and ordered Mn moment $m_{0}=m_{\text {ord }}(T \rightarrow 0)=1.8(1) \quad \mu_{\mathrm{B}}$ [14], whereas the strong spin-orbit coupling results in the shortest helix pitch ( $29 \AA$ at low temperature) of the B20 series [15]. The giant topological Hall effect (THE) and the Nernst effect $[16,17]$ make MnGe promising for spintronic applications. In bulk MnGe in a zero field, a helical multidomain ground state was inferred from magnetic neutron diffraction, although a more complex ground state involving a cubic lattice of skyrmions and antiskyrmions was also proposed to account for the THE [18]. With increasing temperature, a fluctuating inhomogeneous chiral phase settles in, extending over an exceptionally broad temperature range $T_{\mathrm{N}} \pm 70 \mathrm{~K}$. Fluctuations below $T_{\mathrm{N}}$ are a unique feature in the B20 series, where they usually extend over a few degrees above $T_{\mathrm{N}}$.

In MnGe, these fluctuations, revealed by a broad susceptibility peak versus temperature, were studied by neutron 
diffraction and Mössbauer spectroscopy $[19,20]$. These measurements suggest a qualitative picture of the chiral inhomogeneties. Below $T_{\mathrm{N}}$, long-range ordered (LRO) helices coexist with short-range ordered (SRO) fluctuating ones. Above $T_{\mathrm{N}}$, the static LRO helices disappear, but SRO helices remain. Ferromagnetic correlations persist up to about $250 \mathrm{~K}$, with a coherence length below the typical helical wavelength. Lowfield magnetic irreversibilities are seen even above, up to $300 \mathrm{~K}$ at least, showing that some sort of slow dynamics coexists with rapid spin fluctuations deeply in the paramagnetic regime.

The nature and origin of the spin fluctuations in $\mathrm{MnGe}$ and the intrinsic inhomogeneity of its chiral order are a matter of debate. The peculiar band structure of $\mathrm{MnGe}$ yields three possible states for the Mn moment, namely, high spin, low spin, and zero spin (called HS, LS, and ZS, respectively) [21], which can be stabilized depending on the interatomic distance. Therefore, the transition between spin states can be driven by an applied pressure. High-pressure neutron diffraction [14] shows that the collapse of the ordered Mn moment in the ground state occurs in two steps, around $7 \mathrm{GPa}$ and above $13 \mathrm{GPa}$, respectively. X-ray data measured up to $30 \mathrm{GPa}$ [22] suggest that the same scenario is at play for the local moment in the paramagnetic phase at $300 \mathrm{~K}$. Altogether, the pressure data suggest a first-order transition line between the HS and LS states, stabilized in a very large $T$ range. This scenario yields a possible route for unconventional Invar-like spin fluctuations, needed to accommodate HS and LS regions with different specific volumes.

We have investigated MnGe by muon spin resonance $(\mu \mathrm{SR})$. At low temperature $(T=10 \mathrm{~K})$ when the frozen helical order is stabilized, we observe a complex oscillating asymmetry as in $\mathrm{MnSi}$ [23]. Following Ref. [23], we account for it by calculating the distribution of dipolar fields at the muon sites. Our analysis allows us to identify the muon stopping site in good agreement with an ab initio model and to determine the contact field on the muon site. With increasing temperature, we use $\mu \mathrm{SR}$ to probe the spin fluctuations at a longer time scale $\left(10^{-6} \mathrm{~s}\right)$ than the Mössbauer $\left(10^{-8} \mathrm{~s}\right)$ or neutron $\left(10^{-11}\right.$ to $\left.10^{-12} \mathrm{~s}\right)$ probes. We deduce from our results the fluctuating fraction and relaxation rate versus temperature, describing the spin dynamics of the chiral fluctuations over a broad temperature range (10-300 K) and time window. The whole results are discussed with respect to the model $\mathrm{MnSi}$ case.

\section{EXPERIMENTAL DETAILS}

Polycrystalline MnGe was synthesized under $8 \mathrm{GPa}$ in a toroidal high-pressure apparatus by melting reaction with $\mathrm{Mn}$ and Ge. The purity of the constituents was $99.9 \%$ and $99.999 \%$ for $\mathrm{Mn}$ and $\mathrm{Ge}$, respectively. The pellets of well-mixed powdered constituents were placed in rock-salt pipe ampoules and then directly electrically heated to $T \simeq 1600^{\circ} \mathrm{C}$. Then the sample was quenched to room temperature before releasing the applied pressure [13]. The sample was the same as for the neutron experiments of Refs. [14,19]. It was checked by neutron and $\mathrm{x}$-ray diffraction, yielding an amount of impurities smaller than $2 \%$. For the purpose of the $\mu$ SR experiment, it was sintered in a pellet of $13 \mathrm{~mm}$ diameter and $2 \mathrm{~mm}$ thickness, wrapped in a thin $\mathrm{Al}$ foil and placed in a silver sample
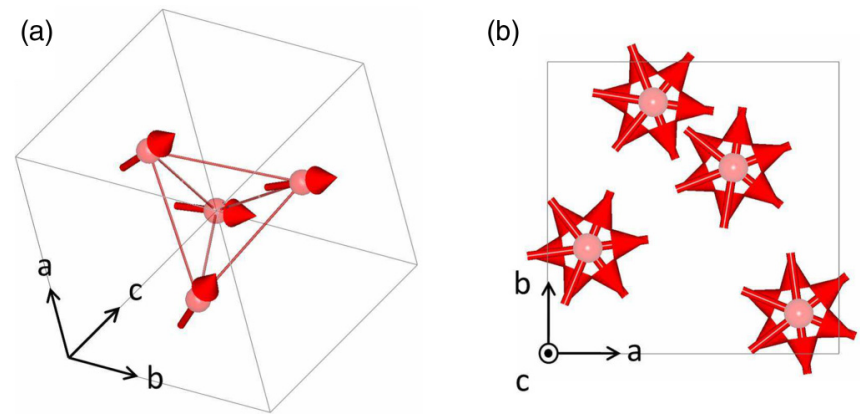

FIG. 1. Magnetic structure of MnGe deduced from neutron powder diffraction as seen along (a) $[1,1, \overline{1}]$ and (b) $[0,0,1]$ crystallographic directions. The latter picture consists of six consecutive unit cells, stacked along the $c$ axis.

holder. The $\mu$ SR experiments were performed on the generalpurpose surface-muon (GPS) instrument at the Paul Scherrer Institut (PSI, Villigen, Switzerland), in the temperature range 10-300 K. In order to study the spin fluctuations, we used longitudinal field $\mu \mathrm{SR}$ (LF- $\mu \mathrm{SR}$ ) in a small field of $20 \mathrm{G}$ to decouple the contribution of nuclear dipolar fields [24]. Measurements at selected temperatures in the range $1.5 \leqslant$ $T \leqslant 115 \mathrm{~K}$ were performed with a shorter time window $(5 \mu \mathrm{s})$ and high statistics to extract the polarization oscillations induced by the helical order. Transverse-field measurements (TF- $\mu \mathrm{SR}$ ) were performed above $170 \mathrm{~K}$ and the frequency shift was compared with the bulk magnetization to evaluate the contact field at the muon site in the paramagnetic region. To measure the magnetization, we used the same sample batch, magnetic field value $(0.4 \mathrm{~T})$, and cooling procedure as for the TF- $\mu$ SR.

\section{RESULTS AND ANALYSIS}

\section{A. Helical order at low temperature}

The helical magnetic structure of $\mathrm{MnGe}$ is shown in Fig 1. The asymmetry of the positron emission, reflecting the time dependence of the muon polarization, recorded at low temperature $(10 \mathrm{~K})$ with high statistics clearly exhibits an oscillating behavior with double frequency (Fig. 2). This oscillatory part of the muon polarization can be associated to the precession of the muon in the field distribution $D\left(B_{\mathrm{loc}}\right)$

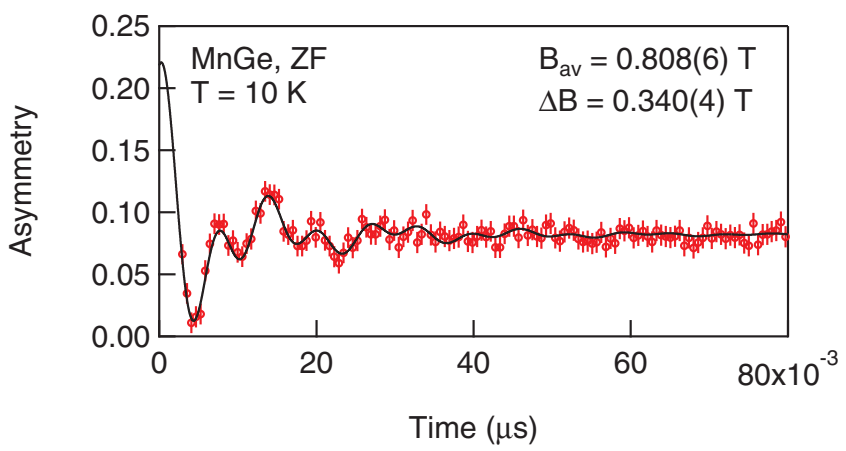

FIG. 2. Time dependence of the asymmetry measured at short times at $T=10 \mathrm{~K}$. The solid curve is a fit of Eq. (2) to the data; see text. 
TABLE I. Coordinates of the muon and its nearest Mn neighbors in the cubic unit cell.

\begin{tabular}{|c|c|c|c|c|c|}
\hline $4 a$ site & & $I$ & $I I$ & III & $I V$ \\
\hline \multirow[t]{2}{*}{ Muon position } & & $\left(x_{\mu}, x_{\mu}, x_{\mu}\right)$ & $\left(\frac{1}{2}-x_{\mu}, \bar{x}_{\mu}, \frac{1}{2}+x_{\mu}\right)$ & $\left(\frac{1}{2}+x_{\mu}, \frac{1}{2}-x_{\mu}, \bar{x}_{\mu}\right)$ & $\left(\bar{x}_{\mu}, \frac{1}{2}+x_{\mu}, \frac{1}{2}-x_{\mu}\right)$ \\
\hline & 1 & $\left(\frac{1}{2}-x, 1-x, \frac{1}{2}+x\right)$ & $(x, x-1, x+1)$ & $(x+1, x, x-1)$ & $(x-1, x+1, x)$ \\
\hline \multirow[t]{2}{*}{ Mn nearest neighbors } & 2 & $\left(\frac{1}{2}+x, \frac{1}{2}-x, 1-x\right)$ & $\left(x-\frac{1}{2}, \bar{x}-\frac{1}{2}, 1-x\right)$ & $\left(\frac{3}{2}-x, \bar{x}, x-\frac{1}{2}\right)$ & $\left(\bar{x}-\frac{1}{2}, 1-x, x-\frac{1}{2}\right)$ \\
\hline & 3 & $\left(1-x, \frac{1}{2}+x, \frac{1}{2}-x\right)$ & $\left(\bar{x}, x-\frac{1}{2}, \frac{3}{2}-x\right)$ & $\left(1-x, x-\frac{1}{2}, \bar{x}-\frac{1}{2}\right)$ & $\left(x-\frac{1}{2}, \frac{3}{2}-x, \bar{x}\right)$ \\
\hline
\end{tabular}

induced by the helical order through

$$
P(t)=\int_{B_{\mathrm{loc}}^{\min }}^{B_{\mathrm{lax}}^{\max }} D\left(B_{\mathrm{loc}}\right)\left[\frac{1}{3}+\frac{2}{3} \cdot \cos \left(\gamma_{\mu} B_{\mathrm{loc}} t\right)\right] d B_{\mathrm{loc}},
$$

where $B_{\mathrm{loc}}^{\min }\left(B_{\mathrm{loc}}^{\max }\right)$ is the minimum (maximum) cutoff field value (see below) and $\gamma_{\mu}=2 \pi \cdot 135.5 \mathrm{MHz} \mathrm{T}^{-1}$ is the muon gyromagnetic ratio.

In a first step, $P(t)$ was fitted by the following analytical expression, similar to that discussed in Eq. (17) of Ref. [23], which catches the essential features of the field distribution sensed by the muon:

$$
\begin{aligned}
P(t) & =\frac{A(t)-b}{A_{0}-b} \\
& =\frac{2}{3} J_{0}\left(\gamma_{\mu} \Delta B t\right) \cos \left(\gamma_{\mu} B_{\mathrm{av}} t+\psi\right) e^{-\lambda_{\mathrm{a}} t}+\frac{1}{3} e^{-\lambda_{\mathrm{b}} t},
\end{aligned}
$$

where $A_{0}=A(t \rightarrow 0)$ is the effective initial asymmetry, $b=$ 0.007 is a temperature-independent background, $J_{0}$ is a Bessel function of the first kind, and $\psi$ is a phase term. $B_{\mathrm{av}}$ and $\Delta B$ are, respectively, the average field and width of the field distribution at the muon site.

The two terms of the sum stem from the powder nature of the sample. Assuming random orientation of the helical domains and of the corresponding fields at the muon sites, on average, two-thirds of the implanted muons precess around a field perpendicular to their spin, whereas one-third of them experience a field along the initial muon polarization and do not precess. The relaxation rates $\lambda_{\mathrm{a}}$ and $\lambda_{\mathrm{b}}$ reflect, as usual, static or dynamic effects, which will be discussed in detail in the next section by considering the full time scale. As in $\mathrm{MnSi}$, the muon is assumed to stop at the Wyckoff position $4 a$ of the space group $P 2{ }_{1} 3: I$, located on the threefold rotation axis and the equivalent $I I, I I I$, and $I V$ linked by this rotation (see Table I). This will be justified below by ab initio calculations. According to neutron diffraction data for $\mathrm{MnGe}$, the helical order propagates along the [001] (or equivalent) direction, and not the [111] direction as found in MnSi. Therefore, in contrast to $\mathrm{MnSi}$ where the muon site $4 a-I$ experiences a narrower field distribution than the others, the four muon sites are magnetically equivalent in $\mathrm{MnGe}$, with each of them feeling basically the same local-field distribution.

The width of the field distribution $\Delta B$ stems from the variation of the Mn moment direction from one cell to another induced by the helical order. A distance equivalent to the helical wavelength, $\lambda_{\mathrm{H}}=2 \pi /|\mathbf{k}|$, where $\mathbf{k}$ is the wave vector of the helix, is necessary to recover the same local magnetic environment at a muon site. It corresponds to about six unit cells for MnGe and 40 unit cells for MnSi.
As shown in Fig. 2, Eq. (2) yields a good fit of the experimental asymmetry. The fast Fourier transform (FFT) of the fitted curve, plotted in Fig. 3, shows the experimental
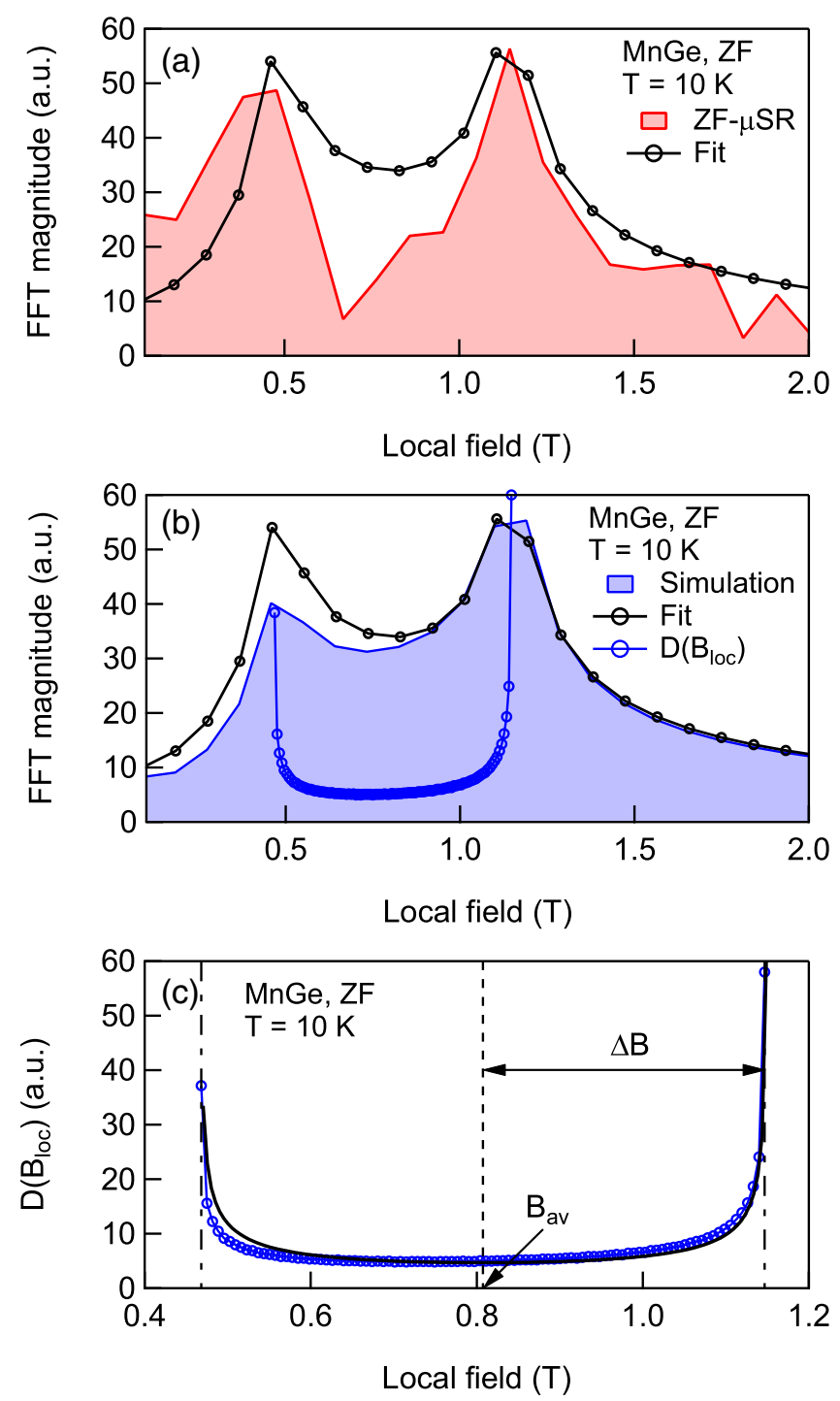

FIG. 3. ZF- $\mu$ SR results for MnGe at 10 K. (a) Fast Fourier transforms (FFTs) of the raw data (red area) and of the fitted curve (black symbols) (see Fig. 2). (b) FFTs of the fitted curve now compared with the FFTs of the simulated signal (blue area) and the calculated local-field distribution $D\left(B_{\text {loc }}\right)$ (blue symbols). (c) Calculated local-field distribution $D\left(B_{\text {loc }}\right)$ (blue symbols). Black line is a fit of a shifted Overhauser function [Eq. (3)] to the data. See text for more details. 
distribution of internal fields deduced from this analysis. Following Ref. [23], this distribution can be approximated by a shifted Overhauser function,

$$
D\left(B_{\mathrm{loc}}\right)=\frac{1}{\pi} \frac{1}{\sqrt{\Delta B^{2}-\left(B_{\mathrm{loc}}-B_{\mathrm{av}}\right)^{2}}},
$$

where $B_{\mathrm{av}}=\left(B_{\max }+B_{\min }\right) / 2$ and $\Delta B=\left(B_{\max }-B_{\min }\right) / 2$, with $B_{\min }$ and $B_{\max }$ the respective minimum and maximum cutoff values of the local-field distribution. The peak width of the experimental field distribution arises from the limited time window of the FFT and the disorder inherent to the magnetic structure.

In a second step, the parameters of the field distribution were refined by performing a numerical calculation of the local field $\mathbf{B}_{\text {loc }}$ at the muon site. The method we used for determining $\mathbf{B}_{\text {loc }}$ in MnGe is explained in full detail in Appendix A. For the sake of simplicity, here we reproduce only the main steps.

For a given $\mathbf{R}_{\mu}$ vector joining the muon site to a Mn ion, the total field is defined as

$$
\mathbf{B}_{\text {loc }}\left(\mathbf{R}_{\mu}\right)=\mathbf{B}_{\text {dip }}\left(\mathbf{R}_{\mu}\right)+\mathbf{B}_{\text {cont }}\left(\mathbf{R}_{\mu}\right),
$$

where $\mathbf{B}_{\text {dip }}$ and $\mathbf{B}_{\text {cont }}$ are the dipolar and contact field, respectively. The dipolar field can be expressed as

$$
\mathbf{B}_{\mathrm{dip}}(\delta)=\mathbf{C}_{\mathrm{dip}} \cdot \cos \delta+\mathbf{S}_{\mathrm{dip}} \cdot \sin \delta,
$$

where the lattice sums $\mathbf{C}_{\text {dip }}$ and $\mathbf{S}_{\text {dip }}$ are performed over a sphere of radius greatly overcoming the helical wavelength (involving typically $10^{6}$ unit cells) and $\delta=2 \pi \mathbf{k} \cdot \mathbf{R}_{\mu}$ can take all values between 0 and $2 \pi$ for an incommensurate structure, simulating all possible local environments along the spin helices for the muon. The contact field $\mathbf{B}_{\text {cont }}$ can be expressed as

$$
\mathbf{B}_{\mathrm{cont}}=\frac{A_{\mathrm{cont}}}{N} \cdot \sum_{i=1}^{N} \mathbf{m}_{\mathbf{i}},
$$

where $A_{\text {cont }}$ is the contact coupling constant, $N=3$ is the number of $\mathrm{Mn}$ ion's nearest neighbors of the muon, and $\mathbf{m}_{\mathbf{i}}$ is their magnetic moment,

$$
\mathbf{m}_{\mathbf{i}}=m_{\mathrm{ord}} \cdot(\cos \varphi \cdot \mathbf{a}-\sin \varphi \cdot \mathbf{b}),
$$

where $m_{\text {ord }}$ is the ordered moment, with $\mathbf{a}$ and $\mathbf{b}$ being unit base vectors of the cubic unit cell and the minus sign accounting for the left handedness of the magnetic spirals of $\mathrm{MnGe}$ [25]. The phase term $\varphi=2 \pi \mathbf{k} \cdot \mathbf{R}_{\mathrm{ij}}$, where $\mathbf{R}_{\mathrm{ij}}$ is a Mn-Mn vector, allows calculating the relative orientation of the Mn moments in the $(\mathbf{a}, \mathbf{b})$ plane, perpendicular to the helical wave vector k. Such an approach yields large canting angle between neighboring $\mathrm{Mn}$ ions in $\mathrm{MnGe}\left(\simeq 30^{\circ}\right.$ between site $4 a-I$ and $\left.4 a-I I\right)$, as expected by the strong spin-orbit coupling, while this angle is extremely small in $\mathrm{MnSi}\left(\simeq 2^{\circ}\right)$. Weak antiferromagnetic modes induced by the Dzyaloshinskii-Moriya interaction, considered theoretically in Refs. [26,27], could lead to small out-of-plane tilts of the magnetic moments. They have not been detected yet by neutron diffraction and their existence would not change the conclusions of this paper.

The harmonic approximation of Eq. (7) leads to the same dependence for the dipolar and contact contributions at the muon site, namely,

$$
\mathbf{B}_{\mathrm{cont}}=\mathbf{C}_{\mathrm{cont}} \cdot \cos \delta+\mathbf{S}_{\mathrm{cont}} \cdot \sin \delta .
$$

Inserting Eq. (7) in Eq. (6) allows one to calculate the vectorial sums $\mathbf{C}_{\text {cont }}$ and $\mathbf{S}_{\text {cont }}$, performed over three $\mathbf{M n}$ near neighbors of the muon site (their coordinates are given in Table I).

Experimentally, the distribution of local fields at the muon site $D\left(B_{\text {loc }}\right)$ can be accessed through the real part of a fast Fourier transform (FFT) of the early-time zero-field $\mu \mathrm{SR}$ (ZF$\mu \mathrm{SR}$ ) signal [see Eq. (1)]. The result obtained at $10 \mathrm{~K}$ is shown in Fig. 3(a) and compared with the FFT of the fit of Eq. (3) to the data.

In order to check the origin of the derived spectrum, mainly composed of two maxima, we have computed the expected $D\left(B_{\text {loc }}\right)$ in MnGe by setting the magnetic and crystal parameters from the neutron data $[14,15]$ measured on the same sample at the same temperature $(T=10 \mathrm{~K})$, namely, the lattice constant $a=4.769 \AA$, the fractional coordinate of the $\mathrm{Mn}$ ions $x=0.138$ r.l.u., the helical wavelength $\lambda_{\mathrm{H}}=$ $28.7 \AA$, and the ordered Mn magnetic moment $m_{\text {ord }}=1.83$ $\mu_{\mathrm{B}}$. For these values, the local-field distribution is calculated by sampling $10^{4}$ values of $\delta$, and optimized by a stepwise variation of the muon site coordinate $x_{\mu}$ and the value of the contact coupling constant $A_{\text {cont }}$. The contact contribution to the total field is found to be larger than the dipolar one and it has opposite sign. On average, we evaluate $B_{\text {cont }}=-1.08 \mathrm{~T}$ and $B_{\text {dip }}=0.27 \mathrm{~T}$.

In Fig. 3(b), we plot the calculated $D\left(B_{\text {loc }}\right)$, which is in excellent agreement with the experimental one. In addition, we display a simulation of the expected ZF- $\mu$ SR signal, generated by using the calculated $D\left(B_{\text {loc }}\right)$ and considering the limited time frame, as well as the observed fast damping of the measured asymmetry induced by the disorder inherent to the magnetic structure $\left[\lambda_{\mathrm{a}}\right.$ term in Eq. (2)]. These combined effects lead to a broadening of the Fourier transform, nicely reproducing the experimental spectrum [Fig. 3(b)].

In summary, we find the same field distribution for the four muon sites, which is quite close to the Overhauser function [Fig. 3(c)]. The agreement between the calculated distribution and the experimental one deduced by FFT of the $\mu$ SR signal is very sensitive to the position of the muon site and the value of the coupling constant. We find only one couple of parameters optimizing both the width and average value for the field distribution (Fig. 4), with values $x_{\mu}=0.543$ and $A_{\text {cont }}=-0.591 \mathrm{~T} \mu_{\mathrm{B}}^{-1}$. The average field and field distribution are, respectively, $B=0.808(6) \mathrm{T}$ and $\Delta B=0.340(5) \mathrm{T}$.

The position of the muon site was estimated independently by $a b$ initio calculation as for $\mathrm{MnSi}$ [28]. Details of the calculations for $\mathrm{MnGe}$ are given in Appendix B. The $6 \times 6 \times 6$ grid used to sample the interstitial space associated with the unit cell volume [Fig. 5(a)] reduces to only one interstitial position that corresponds to a candidate muon site having fractional coordinates $\left(x_{\mu}, x_{\mu}, x_{\mu}\right)$ with $x_{\mu}=0.554$ in the unit cell. This value compares well with the experimental value $x_{\mu}=0.543$ deduced from the analysis of our ZF- $\mu$ SR data. The output of the structural relaxation showing the muon site with respect to the Mn and Ge ones is shown in Fig. 5(b).

Asymmetry patterns, recorded at selected temperatures, have been analyzed with the same procedure [example spectra 


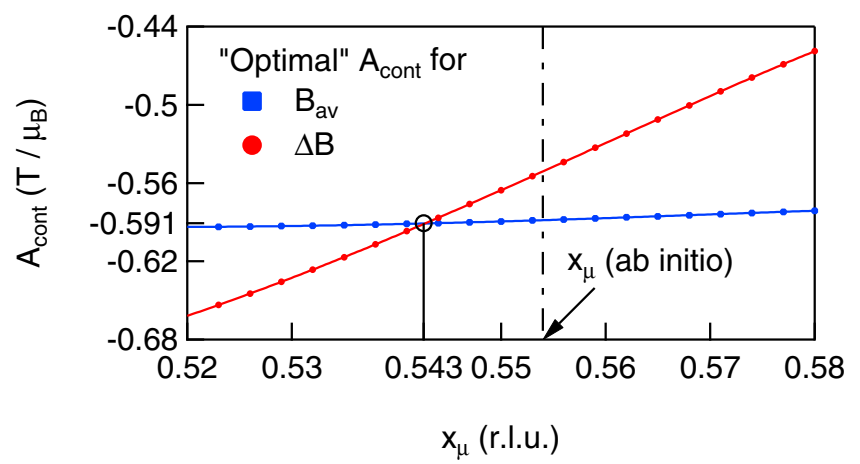

FIG. 4. Determination of the couple of optimized parameters $\left(x_{\mu}\right.$, $\left.A_{\text {cont }}\right)$ : the muon fractional coordinate $x_{\mu}=0.543$ and and the contact coupling constant $A_{\text {cont }}=-0.591 \mathrm{~T} \mu_{\mathrm{B}}^{-1}$ optimize the calculated distribution of the internal field as compared with the one measured at $10 \mathrm{~K}$. See text.

are displayed in Fig. 6(a)]. With increasing temperature, the ordered magnetic moment strongly decreases whereas the propagation vector increases, and these temperature variations are known precisely from neutron diffraction [14,19]. The average field on the muon site is proportional to the ordered Mn moment [Fig. 6(b)], which confirms the validity of the analysis.

\section{B. Spin fluctuations and phase separation}

With increasing temperature, the decrease of the ordered helical moment is associated with the onset of strong spin fluctuations, as MnGe enters the inhomogeneous fluctuating chiral phase. The time dependence of the asymmetry, recorded in a $20 \mathrm{G}$ longitudinal field, is shown in Figs. 7(a) and 7(b) for temperatures above and below $T_{\mathrm{N}}$, respectively. At $10 \mathrm{~K}$, a fast depolarization occurs due to the helical order, so that the effective initial asymmetry $A_{0}=A(t \rightarrow 0)$ drops to one-third of the total asymmetry $A_{\text {tot }}=0.245$, measured well above $T_{\mathrm{N}}$. Upon heating, $A_{0}$ starts increasing around $100 \mathrm{~K}$, when a fluctuating paramagnetic fraction starts coexisting with the ordered one.

In order to account for the phase separation between frozen (LRO) helices and fluctuating (SRO) ones [19], the following functional form was fitted to the long-time tail of the $\mu \mathrm{SR}$
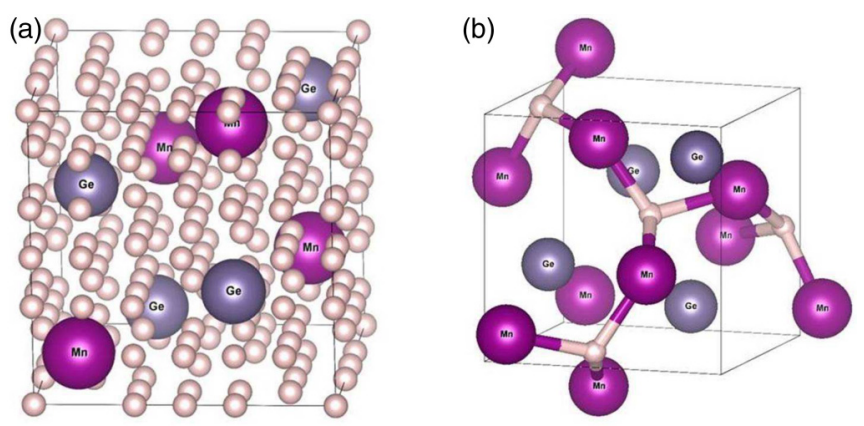

FIG. 5. Result of the $a b$ initio calculation. (a) Interstitial position candidates for a muon site. (b) Final position resulting from the structural relaxation, showing the three Mn neighbors of each muon site.

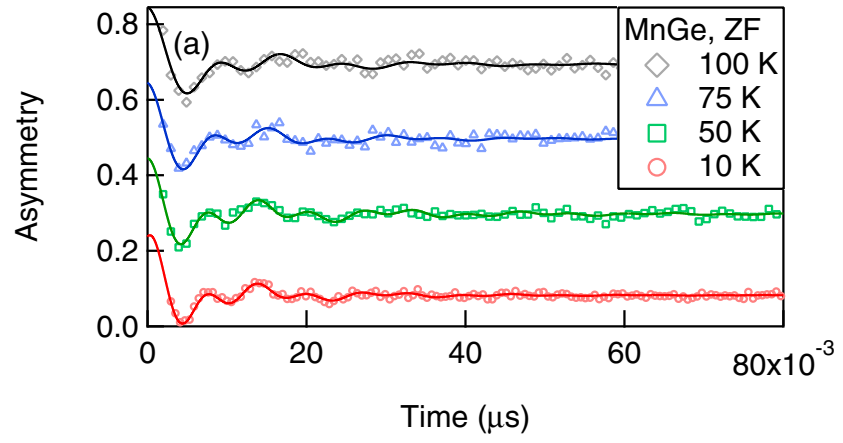

Temperature (K)

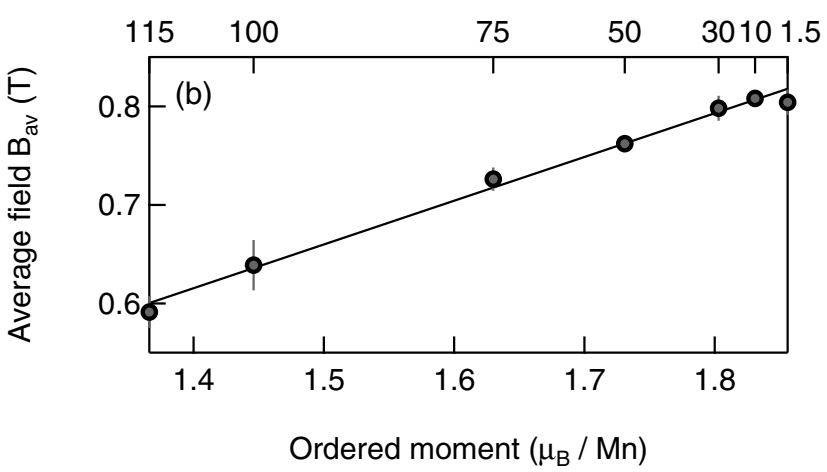

FIG. 6. (a) Asymmetry measured in zero field and at short times for several temperatures. (b) Average internal field $B_{\text {av }}$ compared with the ordered Mn moment measured by neutron diffraction.
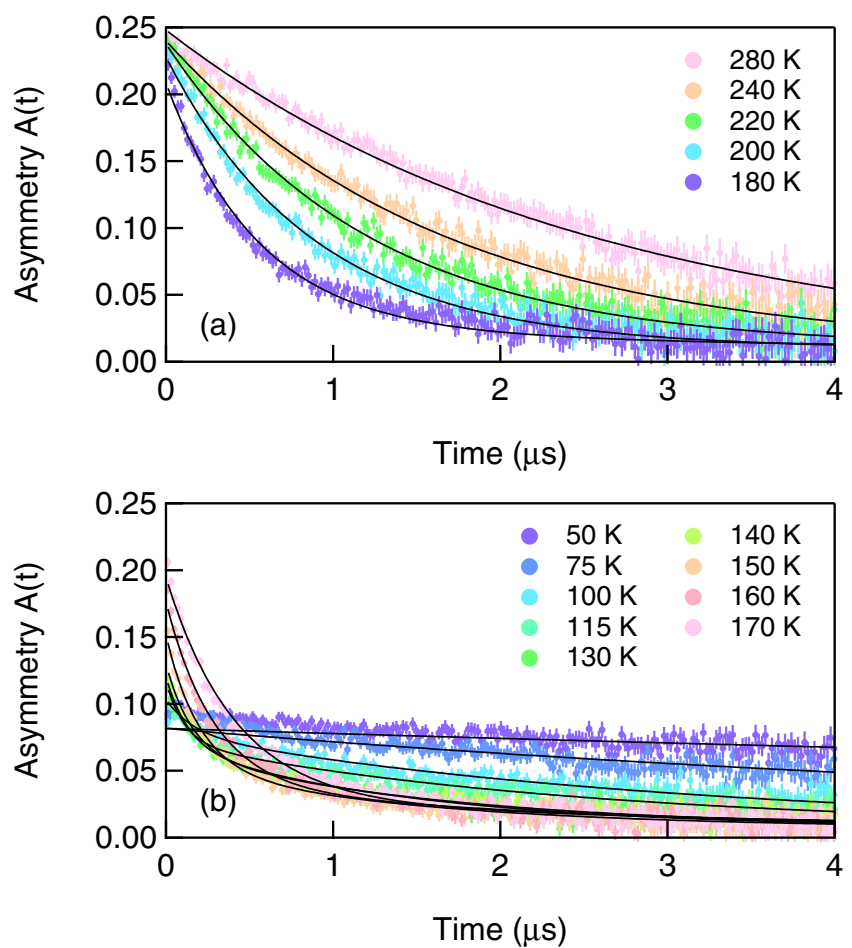

FIG. 7. $\mu$ SR spectra recorded in a $20 \mathrm{G}$ longitudinal field and at selected temperatures (a) above and (b) below $T_{\mathrm{N}}$. Solid curves are fits with Eq. (9), as described in the text. 


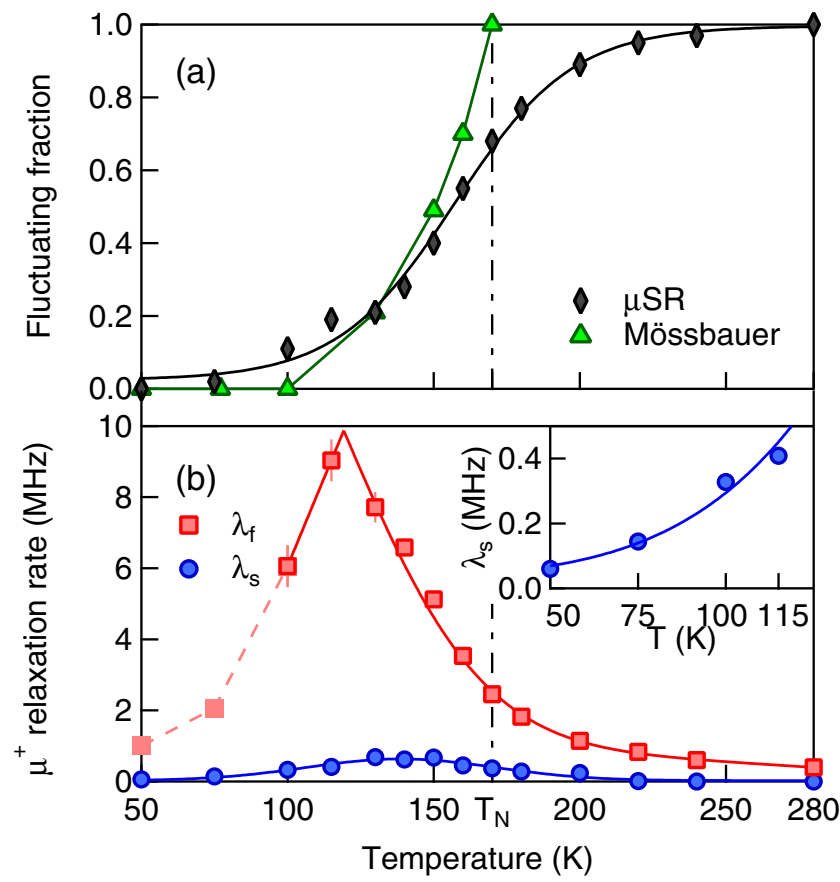

FIG. 8. (a) Fluctuating phase fraction derived from a fit of Eq. (9) to the $\mu \mathrm{SR}$ spectra, in comparison with the paramagnetic fraction deduced from Mössbauer spectroscopy on a ${ }^{57} \mathrm{Fe}$-doped $\mathrm{MnGe}$ sample [19]. (b) Fast and slow relaxation rates obtained through the same analysis. Inset: Slow fluctuations are observed at a small but finite rate $\lambda_{\mathrm{s}}$, down to the lowest temperatures. In all panels, lines are guides to the eye.

$\operatorname{spectra}(t>0.1 \mu \mathrm{s})$ at each temperature:

$$
A(t)=\left(A_{\mathrm{tot}}-b\right)\left[\frac{1-f}{3} e^{-\lambda_{\mathrm{s}} t}+f e^{-\lambda_{\mathrm{f}} t}\right]+b,
$$

where $1-f$ and $f$ are the volume fractions of the long-range ordered and short-range ordered phases, and $\lambda_{s}$ and $\lambda_{f}$ are the corresponding relaxation rates, associated with slow and fast relaxations, respectively. $b$ is a small temperatureindependent background arising from the muons falling into the sample holder, which was measured and subsequently fixed to $7 \times 10^{-3}$. In Eq. (9), one neglects the oscillations at short times $(t<0.1 \mu \mathrm{s})$ discussed in Sec. III A. As shown in Fig. 7, good fits were obtained in the whole temperature range. The temperature dependence of the volume fraction $f$ and relaxation rates $\lambda_{\mathrm{s}}$ and $\lambda_{\mathrm{f}}$ is displayed in Fig. 8. The volume fraction associated with the fast relaxation is in good qualitative agreement with the paramagnetic fraction deduced from published Mössbauer data [19]. Below $100 \mathrm{~K}$, the latter becomes very small ( $f \rightarrow 0$ ) and the associated relaxation rate $\lambda_{\mathrm{f}}$ becomes less reliable. However, the persistence of magnetic fluctuations down to the lowest temperatures is shown by the observation of a finite value for $\lambda_{\mathrm{s}}$ (see inset of Fig. 8).

Instead of a discrete relaxation spectrum composed of two well-defined rates, we alternatively considered a broad distribution of independent relaxation channels, as considered, for instance, in spin glasses [29-31], superparamagnetic particles [32,33], or some frustrated pyrochlore magnets [34-36]. In such case, the asymmetry can be described by a continuous
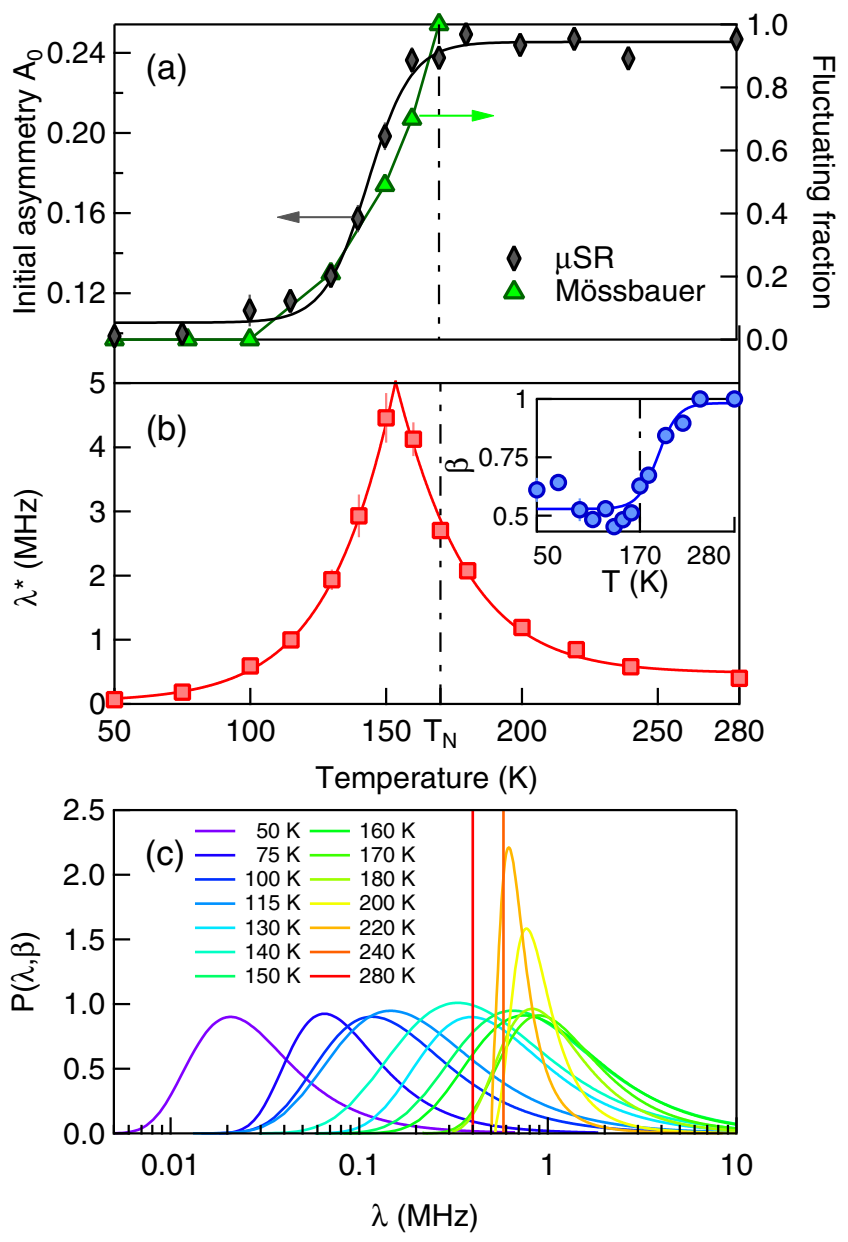

FIG. 9. (a) Effective initial asymmetry of the $\mu \mathrm{SR}$ signal as a function of temperature. The sharp increase of $A_{0}$ upon heating reflects the melting of the long-range helimagnetic order. (b) Characteristic relaxation rate $\lambda^{*}$ obtained from a fit of Eq. (10) to the data (main panel), along with the stretching exponent $\beta$ (inset). (a),(b) Lines are guide to the eye. (c) Calculated relaxation rates distribution computed with the help of experimentally found values for $\lambda^{*}$ and $\beta$ (see text).

sum of exponential decays, usually modeled as a stretched exponential,

$$
A(t)=\left(A_{0}-b\right) e^{-\left(\lambda^{*} t\right)^{\beta}}+b,
$$

where $\lambda^{*}$ is the characteristic muon relaxation rate, $\beta$ is a stretching exponent, and $b$ is defined as explained above. Note that the effective initial asymmetry $A_{0}$ is now refined, as opposed to $A_{\text {tot }}$ which was fixed in Eq. (9). This choice allows focusing on the spin dynamics of the system only by filtering out the fast decay of the measured asymmetry induced by the magnetic order at low temperature. Fitting Eq. (10) to the data in the range $50 \leqslant T \leqslant 280 \mathrm{~K}$ yields an equally good statistical agreement as compared with the two fractions model. The parameters derived from this procedure are displayed in Figs. 9(a) and 9(b).

The thermal evolution of $A_{0}$ is known to be very sensitive to the onset of long-range magnetic order [37]. Indeed, in $\mathrm{MnGe}, A_{0}$ is found to saturate at a value $A_{0}\left(T>T_{\mathrm{N}}\right)=A_{\mathrm{tot}}=$ 
0.245 above $T_{\mathrm{N}}$, and to drop to about one-third of this value in a $70 \mathrm{~K}$ interval below $T_{\mathrm{N}}$, in perfect agreement with the neutron diffraction results of Ref. [19]. In order to describe the overall muon relaxation spectrum described by Eq. (10), we follow the procedure detailed in Ref. [38] where the probability distribution function $P(\lambda)$ is introduced and defined as

$$
\frac{1}{\lambda^{*}} \int_{0}^{\infty} P(\lambda, \beta) e^{-\lambda t} d \lambda=e^{-\left(\lambda^{*} t\right)^{\beta}} .
$$

Since $0.5 \leqslant \beta \leqslant 1$ [see inset of Fig. 9(b)], $\lambda^{*}$ can be regarded as an accurate estimate of the median of $P(\lambda, \beta)$ in the whole temperature range. We thus retain this value as representative of the probed physics and will use it in our evaluation of the electronic spin fluctuation frequencies (Sec. IV). For the sake of completeness, we display calculated $P(\lambda, \beta)$ in Fig. 9(c), allowing one to follow the evolution of the shape of the relaxation spectrum as a function of temperature. While broadened spectra are observed up to about $220 \mathrm{~K}$, a Dirac $\delta$ function-corresponding to a single-frequency spectrum-is recovered at higher temperatures, when magnetic correlations are becoming small with respect to the thermal energy.

As a partial conclusion, we stress that irrespective of the model used to describe the long time LF- $\mu$ SR spectra, our data strongly indicate that spin fluctuations are surviving deep inside the magnetically ordered phase, at odds with other known cubic Dzyaloshinskii-Moriya helimagnets.

\section{Transverse-field measurements in the paramagnetic regime}

In a magnetic field $H$ perpendicular to its initial momentum, the muon spin precesses with a frequency $v$ related to the susceptibility of the bulk material. A typical spectrum measured in $\mathrm{MnGe}$ in the paramagnetic regime with a field of $0.4 \mathrm{~T}$ is shown in the inset of Fig. 10. The frequency shift $v_{0}-v$ with respect to the frequency $v_{0}=54.2 \mathrm{MHz}$ in the $\mathrm{Ag}$ sample holder can be expressed as

$$
v_{0}-v=\frac{\gamma_{\mu}}{2 \pi}\left\langle B_{\mathrm{loc}}\right\rangle
$$

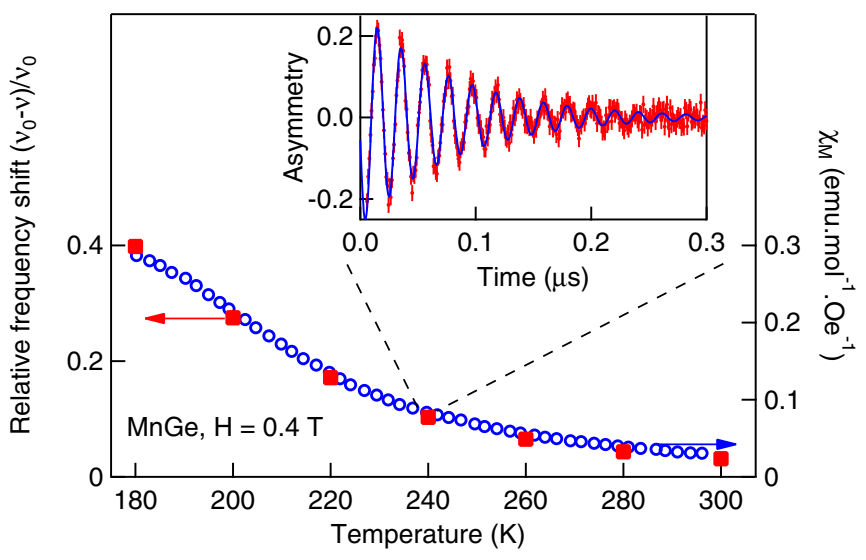

FIG. 10. Relative frequency shift $\left(v_{0}-v\right) / v_{0}$ (red squares, left scale) deduced from TF- $\mu$ SR spectra measured in the paramagnetic regime, in comparison with the macroscopic susceptibility (blue circles, right scale). Both sets of data were measured at $0.4 \mathrm{~T}$. A typical TF- $\mu$ SR spectrum taken at $240 \mathrm{~K}$ is shown in the inset. where the local field

$$
B_{\text {loc }}=B_{\text {ext }}+B_{\text {cont }}+B_{\text {dip }}+B_{\text {Lor }}+B_{\text {dem }}
$$

involves terms corresponding to the applied, contact, dipolar, Lorentz, and demagnetizing fields, respectively, which must be averaged over all orientations. Due to this average, the contribution of the dipolar term cancels, and the contact term reduces to $\mathrm{B}_{\text {cont }}=A_{\text {cont }} \chi_{\mathrm{M}} H$, where $\chi_{\mathrm{M}}$ is the isotropic susceptibility of a $\mathrm{MnGe}$ mole of volume $V_{\mathrm{M}}$, and $\mathrm{A}_{\text {cont }}$ is the isotropic average of the hyperfine contact tensor. The demagnetizing and Lorentz fields are, respectively, equal to $B_{\mathrm{dem}}=-4 \pi N \frac{\chi_{M}}{V_{\mathrm{M}}} H$ and $B_{\text {Lor }}=\frac{4 \pi}{3} \frac{\chi_{\mathrm{M}}}{V_{M}} H$. As shown in Fig. 10, the temperature dependence of the $\mu \mathrm{SR}$ shift compares well with that of the macroscopic susceptibility. Assuming a demagnetization factor corresponding to the shape of the sintered pellet used in our experiment $(N \simeq 0.8)$, we obtain a contact coupling constant $A_{\text {cont }}=-0.45(11) \mathrm{T} \mu_{\mathrm{B}}^{-1}$ from the measured frequency and macroscopic susceptibility $\left(v=48.7 \mathrm{MHz}\right.$ and $\chi_{\mathrm{M}}=$ $8.3 \times 10^{-2} \mathrm{emu} \mathrm{mol}^{-1} \mathrm{Oe}^{-1}$ at $\left.240 \mathrm{~K}\right)$. On the other hand, if we consider a distribution of individual grain shapes within the sample, with $0 \leqslant N \leqslant 1$ in the extreme case, we end up with $A_{\text {cont }}=-0.6(2) \mathrm{T} \mu_{\mathrm{B}}^{-1}$, a value in even closer agreement with our experimental determination by ZF- $\mu \mathrm{SR}$ (see Sec. III A).

\section{DISCUSSION}

The origin of multiple frequency oscillations in $\mu$ SR spectra is usually ascribed to magnetic unequivalent muon stopping sites, as it occurs, for instance, in a $\mathrm{Cu}_{2} \mathrm{OSeO}_{3}$ helical magnet $[39,40]$. An alternative explanation previously proposed in MnSi considers the formation of a spin-polaron state, made by a localized electron state mediating ferromagnetic interactions with the neighboring magnetic ions, and inducing two different states for the muon [41]. Here we can exclude the presence of crystallographic unequivalent muon sites. The two-frequency asymmetry observed in $\mathrm{ZF}-\mu \mathrm{SR}$ is attributed to the field distribution on a given muon site induced by the helical order, and the approach of Ref. [23] in a MnSi single crystal is supported in MnGe with polycrystalline form, shorter helical period, higher moment, and different local symmetry induced by the propagation vector. In $\mathrm{MnGe}$, the magnetic environment of the Mn neighbors is also far from the ferromagnetic droplet required to localize an electron because of the large canting between near-neighbor Mn moments.

In contrast with $\mathrm{MnSi}$ where the muon sitting at the site $4 a \mathrm{I}$ Wickoff site experiences a unique field, in MnGe the muons on the four $4 a$ Wickoff sites feel the same field distribution. The fitted position of the muon site $x_{\mu}=0.543$ compares well with the value $x_{\mu}=0.554$ deduced from the $a b$ initio model. At low temperature $\left(T \ll T_{\mathrm{N}}\right)$, the average field and width of the field distribution in MnGe can be compared with the values relative to the sites $I I, I I I$, and $I V$ in $\mathrm{MnSi}$ (see Table II). The average field is much larger in MnGe than in $\mathrm{MnSi}\left(B_{\mathrm{av}}^{\mathrm{MnGe}} / B_{\mathrm{av}}^{\mathrm{MnSi}} \sim 5\right)$, roughly reflecting the ratio of dipolar contributions $m_{\text {ord }} / a^{3} \sim 4$. On the other hand, the relative widths of the field distributions $\eta=\Delta B / B_{\text {av }}$ are only slightly different, namely, $\eta_{\mathrm{MnGe}} / \eta_{\mathrm{MnSi}} \simeq 1.15$. Indeed, if we would expect measuring a broader local-field distribution for a magnetic structure having a longer periodicity, we must also account for the different ordered moment values. This implies 
TABLE II. Comparison of structural, magnetic, and ZF- $\mu$ SR data for $\mathrm{MnGe}$ and $\mathrm{MnSi}$. In the case of $\mathrm{MnSi}, B_{\mathrm{av}}$ and $\Delta B$ are considered for site II only (see Ref. [23]).

\begin{tabular}{lcc}
\hline \hline & $\operatorname{MnGe}(10 \mathrm{~K})$ & $\operatorname{MnSi}(5 \mathrm{~K})$ \\
\hline$T_{\mathrm{N}}(\mathrm{K})$ & 170 & 29.5 \\
$a(\AA)$ & 4.769 & 4.558 \\
$\lambda_{\mathrm{H}}(\AA)$ & 28.7 & 180 \\
$m_{\text {ord }}\left(\mu_{\mathrm{B}} / \mathrm{Mn}\right)$ & 1.83 & 0.4 \\
$\left|B_{\text {av }}\right|(\mathrm{T})$ & $0.808(6)$ & 0.152 \\
$\Delta B(\mathrm{~T})$ & $0.340(5)$ & 0.056 \\
$\Delta B /\left|B_{\text {av }}\right|$ & 0.42 & 0.37 \\
$A_{\text {cont }}\left(\mathrm{T} \mu_{\mathrm{B}}^{-1}\right)$ & -0.591 & -0.518 \\
$x_{\mu}(\mathrm{r} .1 . \mathrm{u})$ & 0.543 & 0.532 \\
\hline \hline
\end{tabular}

a scaling of the form $\eta \propto 1 /\left(m_{\text {ord }} \cdot \lambda_{\mathrm{H}}\right)$ which, given the material-specific parameters in Table II, yields $\eta_{\mathrm{MnGe}} / \eta_{\mathrm{MnSi}} \simeq$ 1.37 , in agreement with the experimental value.

In both $\mathrm{MnGe}$ and $\mathrm{MnSi}$, the dominant contribution to the local field measured by LF- $\mu$ SR is played by the contact term. As for the contact coupling constants $\mathrm{A}_{\text {cont }}$, we find very similar values in $\mathrm{MnGe}$ and $\mathrm{MnSi}\left(\sim-0.55 \mathrm{~T} \mu_{\mathrm{B}}^{-1}\right)$ from the analysis of the LF- $\mu$ SR spectra measured in the ordered state at low temperature. The value found for MnGe by TF- $\mu$ SR in the applied field in the paramagnetic state $\left(\sim-0.45 \mathrm{~T} \mu_{\mathrm{B}}^{-1}\right)$ is slightly smaller, but entailed by a large error bar due to the uncertainty on the demagnetization factor for a powdered sample.

The time dependence of the asymmetry in the full time window reflects the spin fluctuations in the inhomogeneous chiral phase. Assuming a dynamical phase separation in $\mathrm{MnGe}, \mu \mathrm{SR}$ probes two different electronic relaxation rates for the Mn moments, reflected in typical values of the muon relaxation rates differing by more than an order of magnitude. The phase ratio between the slow and fast fluctuating fractions can be compared with the ratio of "frozen" to "paramagnetic" fractions deduced from Mössbauer spectroscopy. The latter probes a shorter and narrower time window $\left(10^{-7}\right.$ to $\left.10^{-9} \mathrm{~s}\right)$ than $\mu \mathrm{SR}$, but the qualitative agreement is very good. A more phenomonological description, assuming a broad distribution of frequencies with a shape evolving with temperature [as traced by the change of stretching exponent $\beta(T)$ in Fig. 9(b)] is also compatible with the $\mu \mathrm{SR}$ data, and it might explain why in the Mössbauer spectroscopy with narrow time window, one does not observe any relaxing behavior. The plateau value reached by the stretched exponent below $T_{\mathrm{N}}(\beta=0.5)$ has been observed both in dilute spin glasses due to random fields [42] and in geometrically frustrated magnets [36].

Altogether, fast and slow spin fluctuations can coexist over a temperature range extended by the muon probe to about $50-250 \mathrm{~K}$, which is a huge range with respect to other chiral magnets. We relate the slow dynamics to LRO helices and the fast one to SRO helices or ferromagnetic correlations (namely, incomplete helices), recalling that both have been observed, coexisting in the same temperature range, by neutron diffraction [19] and small-angle scattering [20]. We tentatively attribute the origin of this magnetic inhomogeneity in a chemically pure compound to the peculiar MnGe band structure and quenched state, inducing metastable low-spin states in a dominant high-spin state.
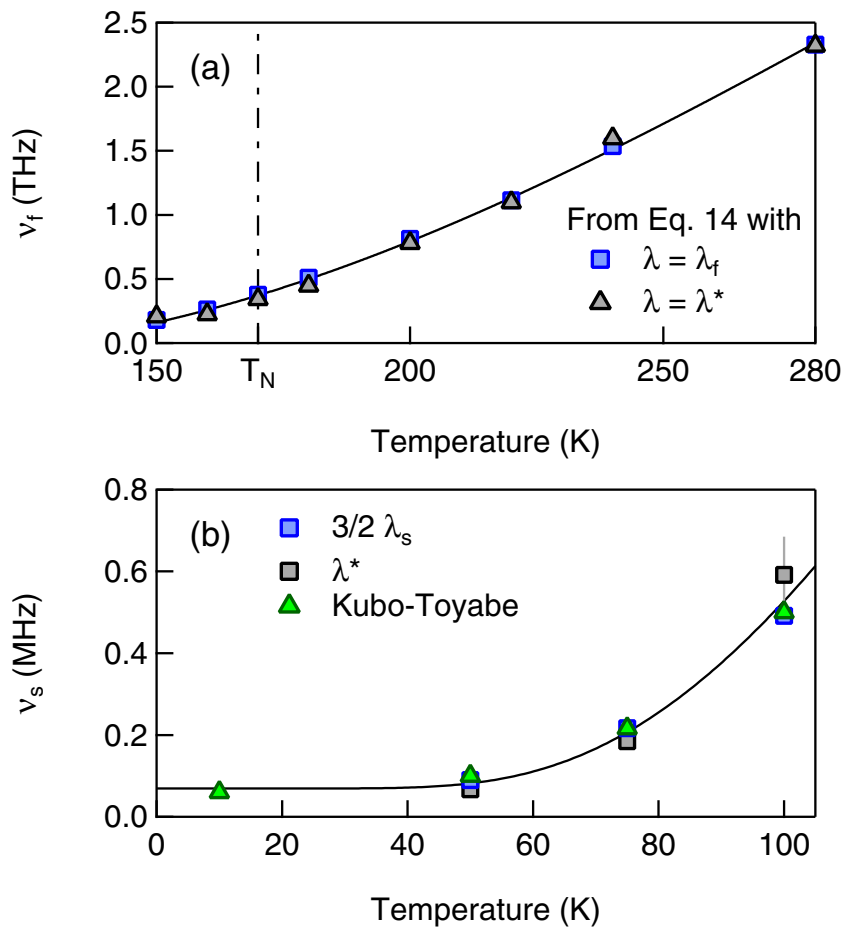

FIG. 11. (a) Thermal evolution of the fast spin fluctuation frequency $v_{\mathrm{f}}$, derived from our LF- $\mu \mathrm{SR}$ measurements by virtue of Eq. (14). (b) Slow fluctuations frequency as a function of temperature obtained in the low-temperature limit. Solid line are guides to the eye.

In the paramagnetic regime, in the limit of fast fluctuations, the muon relaxation rate $\lambda$ is related to the typical electronic spin fluctuation frequency $\nu_{\mathrm{f}}$ by

$$
\lambda=\frac{2 \gamma_{\mu}^{2}\left\langle B^{2}\right\rangle}{v_{\mathrm{f}}},
$$

where $\left\langle B^{2}\right\rangle$ is the second moment of the distribution of fluctuating field experienced by the muons [24]. In the simplest case, the random fluctuations of the magnetic moments well above $T_{\mathrm{N}}$ yield an average field $\langle B\rangle=0$ and a width of the field distribution $\left\langle B^{2}\right\rangle \simeq B_{\mathrm{av}}^{2}$, where $B_{\mathrm{av}} \simeq 0.8 \mathrm{~T}$ is the internal field measured at low temperature. This yields a typical frequency of the fluctuations $v_{\mathrm{f}} \sim 2.5 \mathrm{THz}$ at $300 \mathrm{~K}$, a frequency range which could be probed, e.g., by inelastic neutron scattering. With decreasing temperature, the increase of $\lambda_{\mathrm{f}}$ [Fig. 8(b)] or $\lambda^{*}$ [Fig. 9(b)] reflects the slowing down of the fluctuations when approaching the transition. The evolution of the fluctuation frequency derived from Eq. (14) is illustrated in Fig. 11. In the paramagnetic regime, $v_{\mathrm{f}}$ is a monotonously increasing function of temperature [Fig. 11(a)]. Below $T_{\mathrm{N}}$, the $\mu \mathrm{SR}$ signal becomes quickly dominated by the growing ordered fraction within the sample. In order to get deeper insight into the critical dynamics of $\mathrm{MnGe}$, one should use space-resolved techniques which allows determining the $Q$ dependence of the relaxation spectrum in contrast to local probes such as $\mu$ SR or Mössbauer, where all length scales contribute to the signal.

Conversely, in the ordered regime at low temperature, the residual fluctuations probed by the muon in an almost static distribution of internal fields can be modeled by the 
dynamical Kubo-Toyabe function which, in the slow hopping limit, extrapolates to [24]

$$
A(t) \sim \frac{A_{\text {tot }}}{3} \exp \left(-2 / 3 v_{\mathrm{s}} t\right)
$$

where $v_{\mathrm{s}}$ is the characteristic spin fluctuation frequency within the ordered phase. Neglecting the oscillations at short times, the long-time tail of the LF- $\mu$ SR spectra indeed show a residual slope with respect to the one-third plateau. Estimating $v_{\mathrm{s}}$ via (i) a direct fit of Eq. (15) to the data, (ii) $\lambda_{\mathrm{s}}\left(=2 / 3 v_{\mathrm{s}}\right)$ in Eq. (9), or (iii) $\lambda^{*}$ in Eq. (10) offers a perfect correspondence, as illustrated in Fig. 11(b). These slow fluctuations, inaccessible to the Mössbauer and neutron probes, could correspond to thermally activated lock-in and unlock-in processes for the helices.

\section{SUMMARY AND CONCLUSION}

Our detailed $\mu$ SR study of a MnGe chiral magnet can be discussed with regards to the model $\mathrm{MnSi}$ case. The distribution of internal fields probed by $\mu \mathrm{SR}$ in $\mathrm{MnGe}$ is qualitatively similar to that in $\mathrm{MnSi}$, and quantitatively explained by its peculiar helical order. The model of Amato et al. [23] developed for MnSi could be checked in another compound with a powdered state, shorter helical pitch, higher Mn moment, and different propagation vector. Moreover, in $\mathrm{MnGe}$, the average internal field could be scaled with the ordered Mn moment measured by neutron diffraction versus temperature. As in $\mathrm{MnSi}$, we identify a unique Wyckoff site for the muon. In both compounds, the analysis supports the helical order as a direct origin of a double-frequency time variation of the asymmetry, without the need to invoke either a spin-polaron state or multiple muon sites. Such analysis could be extended in the future to investigate other helical magnets, providing useful information on systems widely studied for their transport or multiferroic properties.

The $\mu$ SR study also provides information about the spin inhomogeneities in the fluctuating chiral phase, a feature specific to MnGe. These inhomogeneous fluctuations were analyzed in two ways, by considering either a two-phase system or a broad relaxation spectrum, both characterizing the spin dynamics in a large frequency range. Slow and fast spin fluctuations coexist within a large temperature interval, extended by the muon probe to about $50-250 \mathrm{~K}$. This behavior is in stark contrast to $\mathrm{MnSi}$ and other B20 chiral magnets where fluctuations between correlated spins are confined to the transition region, and observed only down to $5 \mathrm{~K}$ below $T_{\mathrm{N}}$ for $\mathrm{MnSi}$. In MnGe, the frequency range of fluctuations probed by $\mu \mathrm{SR}$ extends from the $\mathrm{THz}$ at $300 \mathrm{~K}$ to tens of $\mathrm{kHz}$ at $10 \mathrm{~K}$. The origin of these exceptionally broad temperature range and time scale in $\mathrm{MnGe}$ is still unclear. One reason could be the high value of the DzyaloshinskiiMoryia interaction (DM). Another reason could be the metastable B20 crystal structure and Invar-like character of the MnGe band structure, favoring low-energy fluctuations to accommodate lattice strains between high-spin and low-spin region.

\section{ACKNOWLEDGMENTS}

Part of this work was performed at the Swiss Muon Source (S $\mu$ S, PSI, Villigen, Switzerland). We are grateful to P. Dalmas de Réotier for very useful discussions. The postdoctoral training of N.M. is funded by the LabEx Palm, through a public grant from the "Laboratoire d'Excellence Physics Atom Light Matter" (LabEx PALM) overseen by the French National Research Agency (ANR) as part of the "Investissements d'Avenir" program (Grant No. ANR-10-LABX-0039). The postdoctoral training of M.D. is funded by the ANR (DYMAGE). D.A. acknowledges partial financial support from Romanian UEFISCDI Project No. PN-II-ID-PCE-2011-30583 (85/2011). P.B. thanks the computing resources provided by CINECA within the Scientific Computational Project CINECA ISCRA Class C (Award No. HP10C5EHG5, 2015) and STFC's Scientific Computing Department. L.N.F. and A.V.T. acknowledge the support of the Russian Foundation for Basic Research (Grant No. 14-02-00001). R.D.R. acknowledges financial support provided by the European Union Horizon 2020 research and innovation programme under Grant Agreement No. 654000.

\section{APPENDIX A: DIPOLAR AND CONTACT FIELD CALCULATION}

Here we derive the method used for computing the local field at the muon site (adapted from Refs. [43-45]).

The dipolar field created by an assembly of magnetic moments $\mathbf{m}_{\mathbf{i}}$ at the muon site can be calculated through

$$
\mathbf{B}_{\mathrm{dip}}\left(\mathbf{R}_{\mu}\right)=\frac{\mu_{0}}{4 \pi} \cdot \sum_{\mathrm{i}}\left[\frac{3 \mathbf{r}_{\mathbf{i}}\left(\mathbf{m}_{\mathbf{i}} \cdot \mathbf{r}_{\mathbf{i}}\right)}{\left|\mathbf{r}_{\mathbf{i}}\right|^{5}}-\frac{\mathbf{m}_{\mathbf{i}}}{\left|\mathbf{r}_{\mathbf{i}}\right|^{3}}\right],
$$

where $\mathbf{r}_{\mathbf{i}}=\mathbf{R}_{\mathbf{i}}-\mathbf{R}_{\mu}$ is the vector connecting the $i$ th magnetic moment and the muon. For a helicoidal magnetic structure, $\mathbf{m}_{\mathbf{i}}$ reads

$$
\mathbf{m}_{\mathbf{i}}=m_{\mathrm{ord}} \cdot\left(\cos \varphi_{\mathrm{i}} \cdot \mathbf{a} \pm \sin \varphi_{\mathrm{i}} \cdot \mathbf{b}\right),
$$

where $m_{\text {ord }}$ is the staggered moment, with $\mathbf{a}$ and $\mathbf{b}$ being orthogonal unit vectors in the plane perpendicular to the propagation vector $\mathbf{k}$ of the spin helix. The sign \pm allows for choosing between left- or right-handed spin spirals. In the cubic basis, $\mathbf{a}=(1,0,0)$, $\mathbf{b}=(0,1,0)$, and $\mathbf{k}=\left(0,0, \frac{2 \pi \zeta}{a}\right)$, with $\zeta$ the index of the (incommensurate) magnetic structure and $a$ the cubic lattice constant. The phase term $\varphi_{\mathrm{i}}$ in Eq. (A2) reads

$$
\varphi_{\mathrm{i}}=\mathbf{k} \cdot\left(\mathbf{R}_{\mathbf{i}}-\mathbf{R}_{\mathrm{ref}}\right)=\mathbf{k} \cdot\left(\mathbf{r}_{\mathbf{i}}+\mathbf{R}_{\mu}-\mathbf{R}_{\mathrm{ref}}\right)
$$


where $\mathbf{R}_{\text {ref }}$ is the position of a reference magnetic ion (i.e., for which $\alpha=0$ ). Thus, the cosine and sine terms in (A2) can be rewritten owing to trigonometric identities,

$$
\begin{aligned}
& \mathbf{B}_{\text {dip }}\left(\mathbf{R}_{\mu}\right)=m_{\text {ord }} \cdot \mu_{0} / 4 \pi \\
& \times \cos \left(\mathbf{k} \cdot \mathbf{R}_{\mu}\right)\{\underbrace{\left.\sum_{\mathrm{i}} \cos \left(\mathbf{k} \cdot\left[\mathbf{r}_{\mathbf{i}}-\mathbf{R}_{\mathrm{ref}}\right]\right) \cdot\left[\frac{3 \mathbf{r}_{\mathbf{i}} \cdot\left(\mathbf{a} \cdot \mathbf{r}_{\mathbf{i}}\right)}{\left|\mathbf{r}_{\mathbf{i}}\right|^{5}}-\frac{\mathbf{a}}{\left|\mathbf{r}_{\mathbf{i}}\right|^{3}}\right] \pm \sum_{\mathrm{i}} \sin \left(\mathbf{k} \cdot\left[\mathbf{r}_{\mathbf{i}}-\mathbf{R}_{\mathrm{ref}}\right]\right) \cdot\left[\frac{3 \mathbf{r}_{\mathbf{i}} \cdot\left(\mathbf{b} \cdot \mathbf{r}_{\mathbf{i}}\right)}{\left|\mathbf{r}_{\mathbf{i}}\right|^{5}}-\frac{\mathbf{b}}{\left|\mathbf{r}_{\mathbf{i}}\right|^{3}}\right]\right\}}_{\mathbf{C}_{\mathrm{dip}}}\} \\
& +\sin \left(\mathbf{k} \cdot \mathbf{R}_{\mu}\right)\{\underbrace{\left. \pm \sum_{\mathrm{i}} \sin \left(\mathbf{k} \cdot\left[\mathbf{r}_{\mathbf{i}}-\mathbf{R}_{\mathrm{ref}}\right]\right) \cdot\left[\frac{3 \mathbf{r}_{\mathbf{i}} \cdot\left(\mathbf{a} \cdot \mathbf{r}_{\mathbf{i}}\right)}{\left|\mathbf{r}_{\mathbf{i}}\right|^{5}}-\frac{\mathbf{a}}{\left|\mathbf{r}_{\mathbf{i}}\right|^{3}}\right] \pm \sum_{\mathrm{i}} \cos \left(\mathbf{k} \cdot\left[\mathbf{r}_{\mathbf{i}}-\mathbf{R}_{\mathrm{ref}}\right]\right) \cdot\left[\frac{3 \mathbf{r}_{\mathbf{i}} \cdot\left(\mathbf{b} \cdot \mathbf{r}_{\mathbf{i}}\right)}{\left|\mathbf{r}_{\mathbf{i}}\right|^{5}}-\frac{\mathbf{b}}{\left|\mathbf{r}_{\mathbf{i}}\right|^{3}}\right]\right\}}_{\mathbf{S}_{\mathrm{dip}}}]
\end{aligned}
$$

Owing to the fact that we are dealing with an incommensurate structure, one can replace the argument $\mathbf{k} \cdot \mathbf{R}_{\mu}$ by the continuous variable $\delta$, taking all values between 0 and $2 \pi$. The dipolar field at the muon site eventually reads

$$
\mathbf{B}_{\mathrm{dip}}\left(\mathbf{R}_{\mu}\right)=\mathbf{C}_{\mathrm{dip}} \cdot \cos \delta+\mathbf{S}_{\mathrm{dip}} \cdot \sin \delta .
$$

The advantage in using this form is that the lattice sums $\mathbf{C}_{\text {dip }}$ and $\mathbf{S}_{\text {dip }}$ in Eq. (A4) need to be computed only once. A direct numerical application will converge to better than $0.1 \%$ within a sphere containing more than $\simeq 10^{5}$ unit cells, or even faster by making use of Ewald's summation method.

For computing the contact field, we make use of Eq. (6) from the main text. Using Eq. (A2) for describing the magnetic moments of the three Mn ions nearest neighbors of the muon (see also Table I), we end up with a similar expression as Eq. (A5), namely,

$$
\mathbf{B}_{\text {cont }}\left(\mathbf{R}_{\mu}\right)=\left(\mathbf{C}_{\text {cont }} \cdot \cos \delta+\mathbf{S}_{\mathrm{cont}} \cdot \sin \delta\right),
$$

with

$$
\begin{aligned}
& \mathbf{C}_{\mathrm{cont}}=\frac{A_{\mathrm{cont}} \cdot m_{\mathrm{ord}}}{N}\left\{\sum_{\mathrm{i}=1}^{\mathrm{N}} \cos \left(\mathbf{k} \cdot\left[\mathbf{r}_{\mathbf{i}}-\mathbf{R}_{\mathrm{ref}}\right]\right) \cdot \mathbf{a} \pm \sum_{\mathrm{i}=1}^{\mathrm{N}} \sin \left(\mathbf{k} \cdot\left[\mathbf{r}_{\mathbf{i}}-\mathbf{R}_{\mathrm{ref}}\right]\right) \cdot \mathbf{b}\right\}, \\
& \mathbf{S}_{\mathrm{cont}}=\frac{A_{\mathrm{cont}} \cdot m_{\mathrm{ord}}}{N}\left\{ \pm \sum_{\mathrm{i}=1}^{\mathrm{N}} \sin \left(\mathbf{k} \cdot\left[\mathbf{r}_{\mathbf{i}}-\mathbf{R}_{\mathrm{ref}}\right]\right) \cdot \mathbf{b} \pm \sum_{\mathrm{i}=1}^{\mathrm{N}} \cos \left(\mathbf{k} \cdot\left[\mathbf{r}_{\mathbf{i}}-\mathbf{R}_{\mathrm{ref}}\right]\right) \cdot \mathbf{a}\right\},
\end{aligned}
$$

where $A_{\text {cont }}$ is the contact coupling constant. The sums in Eq. (A7) run over the $N \mathrm{Mn}$ ions, which are the nearest neighbors of the muon. In the case of $\mathrm{MnGe}$, where both muons and Mn ions are sitting on the $4 a$ site of the space group $P 2{ }_{1} 3, N=3$.

Note that $A_{\text {cont }}$ is given in $\mathrm{T} \mu_{\mathrm{B}}^{-1}$ throughout the paper. For comparison with data reported in Ref. [23], it is, however, possible to express it in mol emu ${ }^{-1}$ via $A_{\text {cont }}\left[\mathrm{mol} \mathrm{emu}^{-1}\right]=$ $10^{4} /\left(\mathcal{N}_{\mathrm{A}} \cdot \mu_{\mathrm{B}}\right) \cdot A_{\text {cont }}\left[\mathrm{T} \mu_{\mathrm{B}}^{-1}\right] \simeq 1.791 \cdot A_{\text {cont }}\left[\mathrm{T} \mu_{\mathrm{B}}^{-1}\right]$, where we have used the Avogadro number $\mathcal{N}_{\mathrm{A}}=6.022 \times 10^{23} \mathrm{~mol}^{-1}$ and the Bohr magneton $\mu_{\mathrm{B}}=9.274 \times 10^{-21} \mathrm{emu}$. The factor $10^{4}$ is due to conversion from the SI to the CGS unit system (i.e., $1 \mathrm{~T}=10^{4} \mathrm{G}$ ).

The (total) local field will finally be obtained as

$$
\begin{aligned}
\mathbf{B}_{\text {loc }}\left(\mathbf{R}_{\mu}\right) & =\mathbf{B}_{\text {dip }}\left(\mathbf{R}_{\mu}\right)+\mathbf{B}_{\mathrm{cont}}\left(\mathbf{R}_{\mu}\right) \\
& =\left(\mathbf{C}_{\mathrm{dip}}+\mathbf{C}_{\mathrm{cont}}\right) \cdot \cos \delta+\left(\mathbf{S}_{\mathrm{dip}}+\mathbf{S}_{\mathrm{cont}}\right) \cdot \sin \delta .
\end{aligned}
$$

Note that for our numerical calculations, we have sampled $10^{4}$ values of $\delta$, which results in the distribution displayed in Fig. 3.

\section{APPENDIX B: AB INITIO CALCULATIONS}

We have estimated the electronic structure of MnGe with density functional theory (DFT) as implemented in the ab initio package QUANTUM ESPRESSO [46], which uses a planewave basis set and the pseudopotential approach to remove chemically inactive core electrons from the description. The generalized gradient approximation [47] (GGA) was used to estimate the exchange and correlation potential, and the ultrasoft pseudopotentials [48] of the GBRV library [49] provided an optimal compromise between efficiency and accuracy. The basis set was expanded up to a kinetic energy cutoff of $70 \mathrm{Ry}$ and $60 \mathrm{Ry}$ for the unit cell and the supercell simulations, respectively, and up to $500 \mathrm{Ry}$ for the charge density. The reciprocal space was sampled with a $8 \times 8 \times 8$ Monkhorst- 
Pack (MP) grid [50]. The reciprocal space of the supercells containing 32 formula units (f.u.) was sampled with the Baldereshi point [51] when performing structural relaxations. A $4 \times 4 \times 4$ MP grid was used when analyzing the effect of the impurity on the magnetic properties of the system (vide infra). The relaxed unit cell obtained with these parameters has a lattice constant of $4.763 \AA$, in very good agreement with experimental estimations. DFT simulations also correctly accounted for the high-spin to low-spin transition discussed in Ref. [21]. The magnetic moment per f.u. in the high-spin configuration is $2.02 \mu_{\mathrm{B}}$, while it reduces to about $1 \mu_{\mathrm{B}}$ in the low-spin state, in agreement with previously published results [21].

The identification of the muon site was conducted with the method discussed in Refs. [28,52-54]. The muon was modeled as a hydrogen atom. A supercell containing 32 f.u. was used to identify the candidate muon sites, which are provided by the structural relaxation of the system containing the impurity. In order to sample the interstitial space of the unit cell, as already mentioned in Sec. III, we first set up a $6 \times 6 \times 6$ grid of interstitial positions for the impurity to be used as a starting configuration for the structural relaxation. We later removed the positions that were too close (less than $1 \AA$ ) to one of the atoms of the hosting material and we finally got rid of all symmetry-equivalent initial positions. This eventually led to a set of 14 initial positions for the impurity. The location of the atoms and of the impurity were optimized (keeping the lattice parameter fixed) until forces were lower than $0.5 \times 10^{-3} \mathrm{Ry} / \mathrm{Bohr}$ and the total-energy difference between self-consistent field steps was less than $1 \times 10^{-4} \mathrm{Ry}$. All 14 structural relaxations converged to one symmetry equivalent position, which is described in the text and shown in Fig. 5.

Recent DFT results [55] brought back the attention on the "passivity" of the muon probe, showing that in some peculiar compounds, the muon can conceal the material response as a result of the perturbation introduced by its positive charge. We have verified that this is not the case for MnGe. Indeed, differently from the striking effect of pressure discussed above, in the supercell simulations the muon's perturbation produces detectable structural displacements that, however, are more pronounced for $\mathrm{Ge}$ and are always smaller than $0.12 \AA$ [56]. These small dislocations lead to negligible modifications of the magnetic moment of the $\mathrm{Mn}$ atoms surrounding the muon, confirming the validity of $\mu \mathrm{SR}$ results.

Using the double adiabatic approximation [28], we estimated the muon's ground-state motion energy and the corresponding wave function. We used both Dirichlet and periodic boundary conditions to solve the Schrödinger equation for the muon in the potential obtained from the interpolation of a point cloud surrounding the muon and we obtained, with both approaches, $E_{\mathrm{GS}}=0.57 \mathrm{eV}$. Since the energy barrier between the neighboring muon sites is twice as high, the expected rate for a thermally activated muon diffusion process is negligible, in agreement with the experimental results performed up to room temperature.
[1] T. Moriya and A. Kawabata, J. Phys. Soc. Jpn. 34, 639 (1973).

[2] C. Pfleiderer, D. Reznik, L. Pintschovius, H. v. Lohneysen, M. Garst, and A. Rosch, Nature (London) 427, 227 (2004).

[3] P. Pedrazzini, H. Wilhelm, D. Jaccard, T. Jarlborg, M. Schmidt, M. Hanfland, L. Akselrud, H. Q. Yuan, U. Schwarz, Y. Grin et al., Phys. Rev. Lett. 98, 047204 (2007).

[4] C. Pappas, E. Lelièvre-Berna, P. Falus, P. M. Bentley, E. Moskvin, S. Grigoriev, P. Fouquet, and B. Farago, Phys. Rev. Lett. 102, 197202 (2009).

[5] A. Hamann, D. Lamago, T. Wolf, H. v. Löhneysen, and D. Reznik, Phys. Rev. Lett. 107, 037207 (2011).

[6] U. K. Rößler, A. N. Bogdanov, and C. Pfleiderer, Nature (London) 442, 797 (2006).

[7] H. Wilhelm, M. Baenitz, M. Schmidt, C. Naylor, R. Lortz, U. K. Rößler, A. A. Leonov, and A. N. Bogdanov, J. Phys. Condens. Matter 24, 294204 (2012).

[8] S. Mühlbauer, B. Binz, F. Jonietz, C. Pfleiderer, A. Rosch, A. Neubauer, R. Georgii, and P. Böni, Science 323, 915 (2009).

[9] S. V. Grigoriev, S. V. Maleyev, E. V. Moskvin, V. A. Dyadkin, P. Fouquet, and H. Eckerlebe, Phys. Rev. B 81, 144413 (2010).

[10] M. Janoschek, M. Garst, A. Bauer, P. Krautscheid, R. Georgii, P. Böni, and C. Pfleiderer, Phys. Rev. B 87, 134407 (2013).

[11] S. A. Brazovskii, Sov. Phys. JETP 41, 85 (1975).

[12] X. Z. Yu, Y. Onose, N. Kanazawa, J. H. Park, J. H. Han, Y. Matsui, N. Nagaosa, and Y. Tokura, Nature (London) 465, 901 (2010).

[13] A. Tsvyashchenko, J. Less Common Metals 99, L9 (1984).

[14] M. Deutsch, O. L. Makarova, T. C. Hansen, M. T. FernandezDiaz, V. A. Sidorov, A. V. Tsvyashchenko, L. N. Fomicheva, F.
Porcher, S. Petit, K. Koepernik et al., Phys. Rev. B 89, 180407 (2014).

[15] O. L. Makarova, A. V. Tsvyashchenko, G. Andre, F. Porcher, L. N. Fomicheva, N. Rey, and I. Mirebeau, Phys. Rev. B 85, 205205 (2012).

[16] N. Kanazawa, Y. Onose, T. Arima, D. Okuyama, K. Ohoyama, S. Wakimoto, K. Kakurai, S. Ishiwata, and Y. Tokura, Phys. Rev. Lett. 106, 156603 (2011).

[17] Y. Shiomi, N. Kanazawa, K. Shibata, Y. Onose, and Y. Tokura, Phys. Rev. B 88, 064409 (2013).

[18] N. Kanazawa, J.-H. Kim, D. S. Inosov, J. S. White, N. Egetenmeyer, J. L. Gavilano, S. Ishiwata, Y. Onose, T. Arima, B. Keimer et al., Phys. Rev. B 86, 134425 (2012).

[19] M. Deutsch, P. Bonville, A. V. Tsvyashchenko, L. N. Fomicheva, F. Porcher, F. Damay, S. Petit, and I. Mirebeau, Phys. Rev. B 90, 144401 (2014).

[20] E. Altynbaev, S.-A. Siegfried, V. Dyadkin, E. Moskvin, D. Menzel, A. Heinemann, C. Dewhurst, L. Fomicheva, A. Tsvyashchenko, and S. Grigoriev, Phys. Rev. B 90, 174420 (2014).

[21] U. K. Rössler, J. Phys.: Conf. Ser. 391, 012104 (2012).

[22] N. Martin, I. Mirebeau, M. Deutsch, J.-P. Itié, J.-P. Rueff, U. Rössler, K. Koepernik, L. Fomicheva, and A. Tsvyashchenko, arXiv:1601.05332v1.

[23] A. Amato, P. Dalmas de Réotier, D. Andreica, A. Yaouanc, A. Suter, G. Lapertot, I. M. Pop, E. Morenzoni, P. Bonfà, F. Bernardini et al., Phys. Rev. B 89, 184425 (2014).

[24] R. S. Hayano, Y. J. Uemura, J. Imazato, N. Nishida, T. Yamazaki, and R. Kubo, Phys. Rev. B 20, 850 (1979). 
[25] S. V. Grigoriev, N. M. Potapova, S.-A. Siegfried, V. A. Dyadkin, E. V. Moskvin, V. Dmitriev, D. Menzel, C. D. Dewhurst, D. Chernyshov, R. A. Sadykov et al., Phys. Rev. Lett. 110, 207201 (2013).

[26] V. A. Chizhikov and V. E. Dmitrienko, Phys. Rev. B 85, 014421 (2012).

[27] V. E. Dmitrienko and V. A. Chizhikov, Phys. Rev. Lett. 108, 187203 (2012).

[28] P. Bonfà, F. Sartori, and R. D. Renzi, J. Phys. Chem. C 119, 4278 (2015).

[29] I. A. Campbell, A. Amato, F. N. Gygax, D. Herlach, A. Schenck, R. Cywinski, and S. H. Kilcoyne, Phys. Rev. Lett. 72, 1291 (1994).

[30] A. Keren, P. Mendels, I. A. Campbell, and J. Lord, Phys. Rev. Lett. 77, 1386 (1996).

[31] R. M. Pickup, R. Cywinski, C. Pappas, B. Farago, and P. Fouquet, Phys. Rev. Lett. 102, 097202 (2009).

[32] R. I. Bewley and R. Cywinski, Phys. Rev. B 58, 11544 (1998).

[33] T. J. Jackson, C. Binns, E. M. Forgan, E. Morenzoni, C. Niedermayer, H. Glückler, A. Hofer, H. Luetkens, T. Prokscha, T. M. Riseman et al., J. Phys. Condens. Matter 12, 1399 (2000).

[34] F. Bert, P. Mendels, A. Olariu, N. Blanchard, G. Collin, A. Amato, C. Baines, and A. D. Hillier, Phys. Rev. Lett. 97, 117203 (2006).

[35] P. Dalmas de Réotier, A. Yaouanc, L. Keller, A. Cervellino, B. Roessli, C. Baines, A. Forget, C. Vaju, P. C. M. Gubbens, A. Amato et al., Phys. Rev. Lett. 96, 127202 (2006).

[36] H. Guo, H. Xing, J. Tong, Q. Tao, I. Watanabe, and Z. an Xu, J. Phys. Condens. Matter 26, 436002 (2014).

[37] P. D. de Réotier and A. Yaouanc, J. Phys. Condens. Matter 9, 9113 (1997).

[38] D. C. Johnston, Phys. Rev. B 74, 184430 (2006).

[39] A. Maisuradze, Z. Guguchia, B. Graneli, H. M. Rønnow, H. Berger, and H. Keller, Phys. Rev. B 84, 064433 (2011).
[40] T. Lancaster, R. C. Williams, I. O. Thomas, F. Xiao, F. L. Pratt, S. J. Blundell, J. C. Loudon, T. Hesjedal, S. J. Clark, P. D. Hatton et al., Phys. Rev. B 91, 224408 (2015).

[41] V. G. Storchak, J. H. Brewer, R. L. Lichti, T. A. Lograsso, and D. L. Schlagel, Phys. Rev. B 83, 140404 (2011).

[42] Y. J. Uemura, T. Yamazaki, D. R. Harshman, M. Senba, and E. J. Ansaldo, Phys. Rev. B 31, 546 (1985).

[43] D.-A. Andreica, Ph.D. thesis, ETH Zurich, 2001.

[44] A. Schenck, D. Andreica, F. N. Gygax, and H. R. Ott, Phys. Rev. B 65, 024444 (2001).

[45] A. Schenck, F. N. Gygax, and Y. Ōnuki, Phys. Rev. B 68, 104422 (2003).

[46] P. Giannozzi, S. Baroni, N. Bonini, M. Calandra, R. Car, C. Cavazzoni, D. Ceresoli, G. L. Chiarotti, M. Cococcioni, I. Dabo et al., J. Phys. Condens. Matter 21, 395502 (2009).

[47] J. P. Perdew, K. Burke, and M. Ernzerhof, Phys. Rev. Lett. 77, 3865 (1996).

[48] D. Vanderbilt, Phys. Rev. B 41, 7892 (1990).

[49] K. F. Garrity, J. W. Bennett, K. M. Rabe, and D. Vanderbilt, Comput. Mater. Sci. 81, 446 (2014).

[50] H. J. Monkhorst and J. D. Pack, Phys. Rev. B 13, 5188 (1976).

[51] A. Baldereschi, Phys. Rev. B 7, 5212 (1973).

[52] J. S. Möller, P. Bonfà, D. Ceresoli, F. Bernardini, S. J. Blundell, T. Lancaster, R. D. Renzi, N. Marzari, I. Watanabe, S. Sulaiman et al., Phys. Scr. 88, 068510 (2013).

[53] J. S. Möller, D. Ceresoli, T. Lancaster, N. Marzari, and S. J. Blundell, Phys. Rev. B 87, 121108 (2013).

[54] S. J. Blundell, J. S. Möller, T. Lancaster, P. J. Baker, F. L. Pratt, G. Seber, and P. M. Lahti, Phys. Rev. B 88, 064423 (2013).

[55] F. R. Foronda, F. Lang, J. S. Möller, T. Lancaster, A. T. Boothroyd, F. L. Pratt, S. R. Giblin, D. Prabhakaran, and S. J. Blundell, Phys. Rev. Lett. 114, 017602 (2015).

[56] The displacements obtained from supercell simulations are roughly of the order of magnitude of the accuracy of the calculation. 